OPEN ACCESS

Edited by:

Bathri Narayan Vajravelu,

MCPHS University, United States

Reviewed by:

Praveen Ramakrishnan Geethakumari,

University of Texas Southwestern Medical Center, United States Andre Santos,

Federal University of Rio de Janeiro,

Brazi

Rasmi Nair,

University of Texas Southwestern Medical Center, United States

*Correspondence: Antonio Augusto Fidalgo-Neto aaneto@firjan.com.br

Sergio Noboru Kuriyama

skuriyama@firjan.com.br

Specialty section:

This article was submitted to

Virology,

a section of the journal

Frontiers in Microbiology

Received: 12 August 2021 Accepted: 23 December 2021 Published: 09 February 2022

Citation: Henriques-Santos BM, Farjun B, Corrêa IA, Figueiredo JdB, Fidalgo-Neto AA and Kuriyama SN

(2022) SARS-CoV-2 Variant Determination Through SNP Assays in Samples From Industry Workers

From Rio de Janeiro, Brazil.

Front. Microbiol. 12:757783.

do: 10.3389/fmicb.2021.757783

\section{SARS-CoV-2 Variant Determination Through SNP Assays in Samples From Industry Workers From Rio de Janeiro, Brazil}

\author{
Bianca Monteiro Henriques-Santos'1, Bruna Farjun', Isadora Alonso Corrêa², \\ Janaina de Barros Figueiredo ${ }^{1}$, Antonio Augusto Fidalgo-Neto ${ }^{3 *}$ and \\ Sergio Noboru Kuriyama ${ }^{1 *}$
}

\begin{abstract}
1 SESI Innovation Center for Occupational Health, Industry Federation of the State of Rio de Janeiro, Rio de Janeiro, Brazil, 2 Department of Virology, Paulo de Góes Institute of Microbiology, Federal University of Rio de Janeiro, Rio de Janeiro, Brazil,

${ }^{3}$ SENAI Innovation Institute for Green Chemistry, Industry Federation of the State of Rio de Janeiro, Rio de Janeiro, Brazil
\end{abstract}

Since the first reported case in December 2019, SARS-CoV-2 infections have become a major public health worldwide. Even with the increasing vaccination in several countries and relaxing of social distancing measures, the pandemic remains a threat especially due to the emergence of new SARS-CoV-2 variants. Despite the presence of an enzyme capable of proofreading its genome, high rates of replication provide a source of accumulation of mutations within the viral genome. In this retrospective study, samples from a cohort of industry workers tested by the SESI's COVID-19 mass testing program from September 2020 to May 2021 were analyzed using a mutation panel in order to describe the circulation of currently identified SARS-CoV-2 variants within the samples obtained in Rio de Janeiro State. Our results demonstrated that the variant of interest (VOI) Zeta has been in circulation since October 2020 and reached $87 \%$ of prevalence in February 2021 followed by a decrease due to the emergence of Gamma variant of concern (VOC). Gamma was detected in January 2021 in our studied population, and its prevalence increased during the following months, reaching absolute prevalence within positive samples in May. The Alpha variant was detected only in 4-7\% of samples during March and April while Beta VOC was not detected in our study. Our data agree with sequencing genomic surveillance databases and highlight the importance of continuous mass testing programs and variant detection in order to control viral spread and guide public health measures.

Keywords: SARS-CoV-2, coronavirus, variant, Alpha, Gamma, Zeta, SNP assay

\section{INTRODUCTION}

Mutations are an intrinsic characteristic of virus replication, especially considering RNA viruses, as is the case of SARS-CoV-2 (Almubaid and Hisham, 2021; Lauring and Emma, 2021). However, coronaviruses are less prone to mutations when compared to other RNA viruses due to an acquired enzyme capable of excising erroneous mutagenic nucleotides incorporated by their main 
RNA polymerase, maintaining relative integrity during replication and transcription, despite their unusually large genomes ( $30 \mathrm{~kb}$ ) (Minskaia et al., 2006; Ferron et al., 2018). As natural selection will, in most cases, undermine the effects of arising mutations, in cases of competitive advantages regarding viral replication, transmission, or immunity escape, frequencies tend to increase. On the other hand, mutations that reduce viral fitness tend to be removed from the circulating virus community. Nevertheless, mutation frequencies are also likely to increase or decrease due to genetic drift (Lauring and Emma, 2021).

A virus with one or more new mutations is referred to as the original virus variant and can differ by one or several mutations. As mutations occur constantly, the emergence of new variants is bound to occur during a pandemic (Janik et al., 2021). Numerous SARS-CoV-2 variants have already been documented globally, all sharing one specific mutation called D614G, which became the dominant globally circulating variant after its appearance in the early COVID-19 pandemic (Huang et al., 2021). However, as the pandemic progressed, three variants of interest rapidly became predominant in several countries and have raised particular concerns: Alpha, Beta, and Gamma, which correspond to Pango lineages B.1.1.7, B.1.351, and P.1, respectively (Burki, 2021). Moreover, several variants of interest have raised awareness, among which is Zeta (Pango lineage P.2).

The SESI Innovation Center for Occupational Health (Rio de Janeiro, Brazil), of the Industry Federation of the State of Rio de Janeiro (Firjan), proposed a mass testing program for SARSCoV-2 targeting the industry worker population of the state of Rio de Janeiro. The program focused on enabling a safe return to economic activity through early identification of infection and mitigation of virus spread in the work environment. The objective of this study was to analyze and describe the circulation of currently identified SARS-CoV-2 variants within the samples obtained in Rio de Janeiro State.

\section{MATERIALS AND METHODS}

Nasopharyngeal samples were collected from industry workers by SESI Innovation Center for Occupational Health (FIRJAN, Rio de Janeiro, Brazil)-trained nurses, as part of the COVID-19 mass testing program in the state of Rio de Janeiro. After collection, samples were maintained at $4^{\circ} \mathrm{C}$ in $2 \mathrm{ml}$ of Dulbecco's Modified Eagle Medium (DMEM) through initial processing.

RNA extractions were performed using Absolutely Total RNA Purification Kit (Ref. 400793, Agilent Technologies) using Agilent Technologies Bravo Automated Liquid Handling Platform, following the manufacturer's protocols. Total RNA was stored at $-80^{\circ} \mathrm{C}$ until further use.

After initial screening through RT-qPCR (CDC, 2020), positive samples for SARS-CoV-2 were randomly selected and a retrograde analysis was performed for mutation and variant characterization. A total of 351 samples were analyzed, spanning 45 samples from each month from November 2020 through May 2021, and 18 samples from September and October each, due to a lower positive sample pool in these 2 months. For this, we used the customizable TaqMan ${ }^{\circledR}$ SARS-CoV-2
Mutation Panel SNP genotyping assay (Ref. 4332077, Applied Biosystems), and TaqPath ${ }^{\text {TM }}$ 1-Step RT-qPCR Master Mix (Ref.: A15300, Applied Biosystems), following the manufacturer's protocols for reaction volumes and cycling conditions. For targeted mutations, see Table 1. Samples identified as wild type correspond to hCoV-19/Wuhan/WIV04/2019 (accession number EPI ISL 402124).

Characterization was done as follows:

- Alpha variant is characterized as positive for DEL69/70, $\mathrm{N} 501 \mathrm{Y}$, and $\mathrm{P} 681 \mathrm{H}$. Beta variant is characterized as positive for N501Y, E484K, and K417N.

- Gamma variant is characterized as positive for N501Y, $\mathrm{E} 484 \mathrm{~K}$, and $\mathrm{K} 417 \mathrm{~T}$. Zeta variant is characterized as positive only for E484K.

- Negative samples for all six SNP mutations were assigned as wild type.

As a positive control, previously sequenced Alpha, Gamma, and Zeta variant samples were also reanalyzed using the SNP genotyping kit for assay validation. We used one control sample for each variant lineage tested, namely, Gamma (accession number EPI_ISL_1060902; Faria et al., 2021) and Zeta (BioProject accession number PRJNA686081; Voloch et al., 2021). Alpha sequence has not been deposited yet for lack of sequencing quality.

The present study was approved by the National Committee of Research Ethics and by the Ethics Committee of Hospital Universitário Clementino Fraga Filho, protocol number 4,317,270, which waives the need for the term of informed consent.

\section{RESULTS AND DISCUSSION}

Rio de Janeiro has over 580,000 industry workers, from which 68,000 individuals were tested by the SESI's COVID-19 mass testing program by the end of June 2021. Of these, none were hospitalized or showed severe symptoms at sample collection, which differentiates the presented data from clinical patient data, and is more directly extrapolatable to general population infection behavior and rates. No worker had clinical indications for testing, and tests were performed as a preventive initiative.

Due to the specific characteristics of the slice of the population reached by the program, most individuals were men, representing $72 \%$ of the samples analyzed in this study. Furthermore, selected samples for variant characterization were from individuals of legal economically active age (mainly 18-60 years old) with a Bayesian distribution (Supplementary Figure 1), mostly between 30 and 40 years old, constituting approximately $40 \%$ of total analyzed samples. The selected sample pool is representative of the total population tested, with no statistically significant difference ( $p=0.6442$, Chi-square test). Overall positive case numbers oscillated during this window, with December being the month with the highest prevalence, with a rate of $5.44 \%$.

SARS-CoV-2 variant emergence surveillance is predominantly influenced by mutations in the spike glycoprotein, which is responsible for the binding to human cells and the major target 
TABLE 1 | SNP genotyping assay targeted mutations, lineage association, gene location, and reference ID.

\begin{tabular}{|c|c|c|c|c|c|c|}
\hline Mutation & Alpha (United Kingdom, B.1.1.7) & Beta (South Africa, B.1.351) & Gamma (Brazil, P.1) & Zeta (Brazil, P.2) & Gene & Assay ID \\
\hline DEL69/70 & $x$ & & & & S & AN9HXTM \\
\hline N501Y & $x$ & $x$ & $x$ & & S & ANPRYZA \\
\hline E484K & & $x$ & $x$ & $x$ & S & ANU7GMZ \\
\hline K417N & & $x$ & & & S & ANZTTXP \\
\hline K417T & & & $x$ & & S & AN49ARF \\
\hline $\mathrm{P} 681 \mathrm{H}$ & $x$ & & & & $S$ & ANCFHV6 \\
\hline
\end{tabular}

TABLE 2 | Variant identification in previously sequenced SARS-CoV-2 samples using SNP assay panel.

\begin{tabular}{|c|c|c|c|c|c|c|c|}
\hline & \multirow[t]{2}{*}{ Mutation } & \multicolumn{2}{|c|}{ UK 902 \#2 } & \multicolumn{2}{|c|}{ P1 USP } & \multicolumn{2}{|c|}{ P2 814} \\
\hline & & Cq WILD & Cq MUT & Cq WILD & Cq MUT & Cq WILD & Cq MUT \\
\hline \multirow[t]{6}{*}{ RT-qPCR result } & DEL69/70 & No Cq & 18.140 & 12.904 & No Cq & 24.924 & No Cq \\
\hline & N501Y & No Cq & 18.883 & No Cq & 11.904 & 25.513 & 33.854 \\
\hline & E484K & 19.773 & No Cq & 17.882 & 12.656 & 24.068 & 19.141 \\
\hline & $\mathrm{K} 417 \mathrm{~N}$ & 20.064 & No Cq & 17.280 & No Cq & 25.834 & No Cq \\
\hline & $\mathrm{K} 417 \mathrm{~T}$ & 20.731 & 27.465 & 23.290 & 12.671 & 25.733 & 33.268 \\
\hline & $\mathrm{P} 681 \mathrm{H}$ & No Cq & 18.950 & 13.039 & No Cq & 23.931 & No $\mathrm{Cq}$ \\
\hline \multirow[t]{7}{*}{ Call } & DEL69/70 & \multicolumn{2}{|c|}{ MUTANT } & \multicolumn{2}{|c|}{ WILD } & \multicolumn{2}{|c|}{ WILD } \\
\hline & N501Y & \multicolumn{2}{|c|}{ MUTANT } & \multicolumn{2}{|c|}{ MUTANT } & \multicolumn{2}{|c|}{ WILD } \\
\hline & E484K & \multicolumn{2}{|c|}{ WILD } & \multicolumn{2}{|c|}{ MUTANT } & \multicolumn{2}{|c|}{ MUTANT } \\
\hline & $\mathrm{K} 417 \mathrm{~N}$ & \multicolumn{2}{|c|}{ WILD } & \multicolumn{2}{|c|}{ WILD } & \multicolumn{2}{|c|}{ WILD } \\
\hline & $\mathrm{K} 417 \mathrm{~T}$ & \multicolumn{2}{|c|}{ WILD } & \multicolumn{2}{|c|}{ MUTANT } & \multicolumn{2}{|c|}{ WILD } \\
\hline & P681H & \multicolumn{2}{|c|}{ MUTANT } & \multicolumn{2}{|c|}{ WILD } & \multicolumn{2}{|c|}{ WILD } \\
\hline & Variant & \multicolumn{2}{|c|}{ Alpha } & \multicolumn{2}{|c|}{ Gamma } & \multicolumn{2}{|c|}{ Zeta } \\
\hline
\end{tabular}

of neutralizing antibodies. Mutations on the receptor-binding domain can modulate interactions with host angiotensinconverting enzyme II (ACE2) facilitating cell entry (Ozono et al., 2021), as is the case of D614G, present in all current variants, and N501Y, a common mutation present in variants of concern (VOCs) Alpha, Gamma, and Beta. This mutation led to lower binding free energy requirement, as well as an increased number of electrostatic interactions with ACE2 (Khan et al., 2021; Ortega et al., 2021). Spike antigenic evolution can also affect antibody immunity and vaccination efficacy since coronaviruses' antigen modification can lead to immune system evasion (Eguia et al., 2021).

This is of special concern given that current approved vaccines tend to prevent disease instead of infection. Spreading events can alter the course of an epidemic and, therefore, prevention and detection should be prioritized. Low vaccination rates along with the drop of population adherence to sanitary measures over time, as is the case of Brazil, leads to continuous viral spread and replication, which, in turn, can also lead to new mutations and variants emergence. Novel strains can also lead to reinfection as well as new infection waves (He et al., 2021; Kirby, 2021; Resende et al., 2021). Furthermore, this could threaten the already strained healthcare resources leading to another public health emergency, requiring extended and more rigorous implementation of distancing strategies (Volz et al., 2021).

In this scenario, our results evidenced the need for constant surveillance and spread mitigation, especially in the face of new
VOCs, which are characterized by increased transmissibility, virulence, aside from decreased effectiveness of public health and social measures or available diagnostics, vaccines, and therapeutics (WHO, 2021).

Considering the likeliness of the emergence of new variants and lineages carrying different mutations during virus replication, even in the presence of an error-correcting enzyme, we assessed the characterization of lineage circulation in the samples received by the program through a retrospective analysis. Validation was carried out using samples that had been previously sequenced and identified. Supplementary Tables 1, 2 show, respectively, nucleotide and amino acid sequences of the control samples. The SNP panel was able to correctly identify each lineage through the combination of specific mutations (Table 2). After initial validation, randomly selected samples were also characterized, and the resulting pattern can be seen in Figure 1.

Within the sample pool analyzed in this study, the Zeta variant was firstly identified in October 2020, in one-third of positive individuals, along with a majority of samples identified as wild type. From November through February, Zeta variant predominance gradually escalated and reached $87 \%$ in the latter. Prevalence retreated to $11 \%$, in March, with the rise of Gamma variant cases. We were unable to identify new cases of the Zeta variant by April.

Despite being firstly characterized as a new variant of interest (VOI) only in March 2021, sequencing retro analyses and 


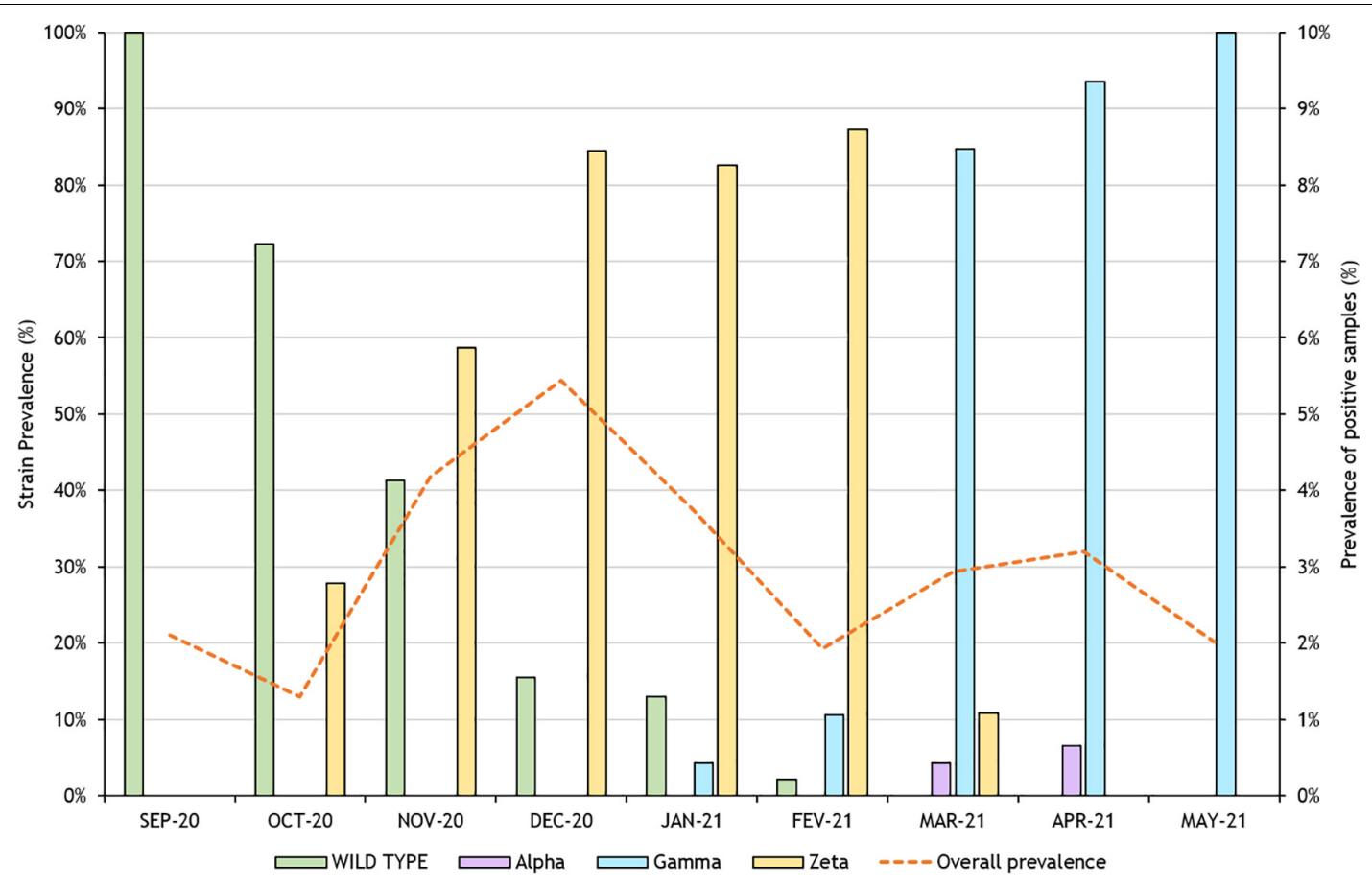

FIGURE 1 | Succession pattern of variants in samples from industry workers from Rio de Janeiro in the months between September 2020 and May 2021. Analyzed variants were Alpha, Beta, and Zeta.

genomic characterization were able to trace this lineage as far back as July 2020, in the state of Rio de Janeiro (Voloch et al., 2021). The Zeta variant continues to be considered a VOI, since its genome has mutations with established or suspected phenotypic implications, while it has been reported to cause community transmission and multiple cases (WHO, 2021). Furthermore, the Zeta variant has been associated with reinfection cases (Nonaka et al., 2021; Resende et al., 2021). This variant displays the E484K spike mutation, shared with both Beta and Gamma variants, an alteration located in the antibody binding site, directly associated with escape from neutralizing antibodies (Hoffmann et al., 2021).

Gamma variants were originally described in travelers from Amazonas, Brazil, to Japan on January 2, 2021 (Fujino et al., 2021). We were able to identify the first individuals with this variant strain by the end of January in samples from Rio de Janeiro, representing $4 \%$ of the total samples analyzed for this month. This quick spread across the country demonstrates little to no control of the virus dissemination and pandemic advance. Prevalence of Gamma variants quickly became predominant among our samples; cases jumped from 11\% in February to 85\% in March, reaching $100 \%$ by May. In Alagoas State, this VOC was initially diagnosed in a patient traveling from Amazonas State by mid-February, along with a case of community transmission (da Silva et al., 2021). Meanwhile, in Rio de Janeiro, there was apparently at least 13 independent events of introduction coming from nearly all regions of the country (Moreira et al., 2021b).

Meanwhile, Alpha variant's original report dates to September 2020 and quickly became the dominant circulating SARS-CoV-2 variant in England (Public Health England [PHE], 2020). In the United States, Alpha was the dominant SARSCoV-2 lineage, representing approximately $60 \%$ of total cases as of the beginning of May, with Delta and Gamma representing approximately $10 \%$ each (CDC, 2021). This VOC was retroactively identified in samples from October 2020 obtained from individuals from São Paulo, Brazil, and local transmission was also reported in the same federative unit, after the identification of the strain in a patient who reported no travel outside of Brazil (Claro et al., 2021; Moreira et al., 2021a). Notwithstanding, among the analyzed samples for this study, the United Kingdom variant comprised only 4 and 7\% in March and April, respectively.

SARS-CoV-2 genomic data gathered by FIOCRUZ corroborates our findings, although the database comprises a different populational segment, with major sequences originating from clinical patients (Fiocruz, 2021). Prevalence profiles show great similarities in the pattern of variant succession in this time window. Considering the regionalized Southeastern compilation of lineage frequencies and the targeted variants here, the overall profile is comparable to the data produced by the present study. Additionally, April and May registered the emergence of a new variant labeled as P.4, Pango lineage, with a prevalence of 1 and 25\%, respectively. This lineage has been identified in São Paulo State so far, but lacks characterization yet, and has been classified neither as a VOC nor as a VOI yet.

Although, during the pandemic, Brazil remained among the highest-ranking countries regarding the number of deaths 
and active cases, diagnosis for SARS-CoV-2 performed by RT-qPCR corresponded so far to a total of 55,034,721 samples, which equates to only 256,953 tests per million inhabitants (WorldOMeter, 2021). In this way, compared to other densely populated countries, Brazil continues to be one of the most affected by the virus, but also one with the fewer tests performed. Therefore, the number of cases might be highly under-reported. In fact, based on extrapolations using both serological surveys and the increase in registered deaths caused by severe acute respiratory infection, along with the increased rate of pneumonia and respiratory insufficiency in 2020, the real number of infected people could be as high as about six times greater than the number notified to the Ministry of Health (Alves et al., 2020; Hallal et al., 2020). Furthermore, as of the end of July 2021 , in 7 months of the program, only approximately $19 \%$ of the total Brazilian population have been fully vaccinated, and $48 \%$ have received at least one dose of the vaccine (OurWorldinData, 2021).

\section{CONCLUSION}

In conclusion, our analysis of positive samples of the 9 months between September 2020 and May 2021 describes the dynamics of the rise of two different variants among the health industry workers from Rio de Janeiro. The rapid escalation of prevalence shown demonstrates that palliation of virus dissemination is primordial to the containment of further new variants that could impair immunization and threaten healthcare. Constant vigilance through sequencing should also be prioritized, aiming to quickly identify frequent mutation in one locus, indicating the possible emergence of a lineage, along with social distancing measures. This could prevent outbreaks of new VOCs, as well as the extension of the pandemic state with the continuous emergence of waves of infected individuals.

\section{REFERENCES}

Almubaid, Z., and Hisham, A. (2021). "Analysis and Comparison of Genetic Variants and Mutations of the Novel Coronavirus SARS-CoV-2.”. Gene Rep. 23:101064. doi: 10.1016/j.genrep.2021.101064

Alves, T. H. E., Tafarel, A. S., Samyla, A. S., Nayani, A. R., and Stefan, V. O. (2020). "Underreporting of Death by COVID-19 in Brazil's Second Most Populous State.”. Front. Public Health 8, 1-7. doi: 10.3389/fpubh.2020.578645

Burki, T. (2021). “Understanding Variants of SARS-CoV-2.”. Lancet 397:462. doi: 10.1016/S0140-6736(21)00298-1

CDC (2021). Variant Proportions." COVID Data Tracker. 2021. Available online at: https://covid.cdc.gov/covid-data-tracker/\#variant-proportions (accessed August 2 2021).

CDC (2020). Real-Time RT-PCR Diagnostic Panel for Emergency Use Only. CDC EUA, Vol. 3. 1-42. Available online at: https://www.fda.gov/media/134922/ download (accessed July 10, 2021)

Claro, I. M., Flavia, C. S. S., Mariana, S. R., Darlan, S. C., Camila, A. M. S., Jaqueline, G. J., et al. (2021). "Local Transmission of SARS-CoV-2 Lineage B.1.1.7, Brazil, December 2020.”. Emerg. Infect. Dis. 27, 970-972. doi: 10.3201/eid2703.210038

Eguia, R. T., Katharine, H. D. C., Terry, S. A., Laurel, K.-M., Alexander, L. G., Janet, A. E., et al. (2021). "A Human Coronavirus Evolves Antigenically to Escape Antibody Immunity.”. PLoS Pathog. 17:1-28. doi: 10.1371/journal.ppat. 1009453

\section{DATA AVAILABILITY STATEMENT}

The raw data supporting the conclusions of this article will be made available by the authors, without undue reservation.

\section{ETHICS STATEMENT}

The studies involving human participants were reviewed and approved by Ethics Committee of Hospital Universitário Clementino Fraga Filho. The Ethics Committee also approved the informed consent waiver.

\section{AUTHOR CONTRIBUTIONS}

$\mathrm{BH}-\mathrm{S}, \mathrm{BF}$, and SK designed and planned the study. BH-S carried out the experiments. JF prepared the samples. BH-S wrote the manuscript with support from BF, IC, and JF. AF-N helped supervise the project. SK and AF-N conceived the original idea and supervised the project. All authors contributed to the article and approved the submitted version.

\section{FUNDING}

This study was funded by the Industry Federation of Rio de Janeiro (FIRJAN).

\section{SUPPLEMENTARY MATERIAL}

The Supplementary Material for this article can be found online at: https://www.frontiersin.org/articles/10.3389/fmicb. 2021.757783/full\#supplementary-material

Faria, N. R., Thomas, A. M., Charles, W., Ingra, M. C., Darlan, S. C., Swapnil, M., et al. (2021). "Genomics and Epidemiology of the P.1 SARS-CoV-2 Lineage in Manaus, Brazil.”. Science 372, 815-821. doi: 10.1126/science.abh 2644

Ferron, F., Lorenzo, S., Ana, T. S. M., Nhung, T. T. L., Marion, S., Laure, G., et al. (2018). "Structural and Molecular Basis of Mismatch Correction and Ribavirin Excision from Coronavirus RNA.”. Proc. Natl. Acad. Sci. 115, E162-E171. doi: 10.1073/pnas.1718806115

Fiocruz, R. G. (2021). Frequência Das Principais Linhagens Do SARS-CoV-2 Por Mês de Amostragem." 2021. Available online at: http://www.genomah cov.fiocruz.br/frequencia-das-principais-linhagens-do-sars-cov-2-por-mes-deamostragem/ (accessed August 2, 2020).

Fujino, T., Hidetoshi, N., Satoshi, K., Mugen, U., Tetsuya, S., Rubuna, S., et al. (2021). "Novel SARS-CoV-2 Variant in Travelers from Brazil to Japan.”. Emerg. Infect. Dis. 27, 1243-1245. doi: 10.3201/eid2704.210138

Hallal, P. C., Bernardo, L. H., Aluísio, J. D. B., Odir, A. D., Fernando, P. H., Lúcia, C. P., et al. (2020). "Evolução Da Prevalência de Infecção Por COVID-19 No Rio Grande Do Sul, Brasil: Inquéritos Sorológicos Seriados.”. Ciência Saúde Col. 25(Suppl. 1), 2395-2401. doi: 10.1590/1413-81232020256.1.09632020

He, D., Guihong, F., Xueying, W., Yingke, L., and Zhihang, P. (2021). The New SARS-CoV-2 Variant and Reinfection in the Resurgence of COVID-19 Outbreaks in Manaus, Brazil. MedRxiv 2021:21254281. doi: 10.1101/2021.03. 25.21254281 
Hoffmann, M., Prerna, A., Rüdiger, G., Alina, S., Bojan, F. H., Alexander, S. H., et al. (2021). SARS-CoV-2 Variants B.1.351 and P.1 Escape from Neutralizing Antibodies. Cell 184, 2384.e-2393.e. doi: 10.1016/j.cell.2021.03.036

Huang, S. W., Sorin, O. M., Chia, H. Y., and Sheng, F. W. (2021). "Impact of Genetic Variability in Ace2 Expression on the Evolutionary Dynamics of SARS-CoV-2 Spike D614G Mutation.”. Genes 12, 1-9. doi: 10.3390/genes120 10016

Janik, E., Marcin, N., Marcin, P., Ireneusz, M., and Michal, B. (2021). "The Emerging Concern and Interest Sars-Cov-2 Variants.”. Pathogens 10, 1-13. doi: 10.3390/pathogens10060633

Khan, A., Tauqir, Z., Muhammad, S., Taimoor, K., Syed, S. A., Aamir, A. A., et al. (2021). Higher Infectivity of the SARS-CoV-2 New Variants Is Associated with K417N/T, E484K, and N501Y Mutants: An Insight from Structural Data. J. Cell. Physiol. 2021, 1-13. doi: 10.1002/jcp.30367

Kirby, T. (2021). "New Variant of SARS-CoV-2 in UK Causes Surge of COVID19.". Lancet Respirat. Med. 9, 20-21.

Lauring, A. S., and Emma, B. H. (2021). "Genetic Variants of SARS-CoV-2 - What Do They Mean?”. JAMA 325, 529-531. doi: 10.1001/jama.2020.27124

Minskaia, E., Tobias, H., Alexander, E. G., Valérie, C., Christian, C., Bruno, C., et al. (2006). "Discovery of an RNA Virus 3'-\&gt;5' Exoribonuclease That Is Critically Involved in Coronavirus RNA Synthesis. Proc. Natl. Acad. Sci. 103, 5108-5113. doi: 10.1073/pnas.0508200103

Moreira, F. R. R., Diego, M. B., Danielle, A. G. Z., Joice, P. S., Aline, B. L., Frederico, S. V. M., et al. (2021a). "Epidemic Spread of SARS-CoV-2 Lineage B.1.1.7 in Brazil.”. Viruses 13:984. doi: 10.3390/v13060984

Moreira, F. R. R., Mirela, D., Diana, M., Alice, L. H., Francine, B. S., Atila, D. R., et al. (2021b). Epidemiological Dynamics of SARS-CoV-2 VOC Gamma in Rio de Janeiro, Brazil. MedRxiv 165, 1-13. doi: 10.1101/2021.07.01.21259404

Nonaka, C. V., Marília, M. F., Tiago, G., Camila, A. L. B., Renata, N. A. M., Karoline, A. F. S., et al. (2021). Genomic Evidence of SARS-CoV-2 Reinfection Involving E484K Spike Mutation, Brazil. Emerg. Infect. Dis. 27, 1522-1524. doi: $10.3201 /$ eid2705.210191

Ortega, J. T., Flor, H. P., Beata, J., and Hector, R. R. (2021). "Mutations in the SarsCov-2 Spike Protein Modulate the Virus Affinity to the Human Ace2 Receptor, an in Silico Analysis.”. EXCLI J. 20, 585-600. doi: 10.17179/excli2021-3471

OurWorldinData (2021). Coronavirus (COVID-19) Vaccinations 2021. Available online at: https://ourworldindata.org/covid-vaccinations?country=OWID_ WRL (accessed July 30, 2021).

Ozono, S., Yanzhao, Z., Hirotaka, O., Kaori, S., Toong, S. T., Kazuo, I., et al. (2021). SARS-CoV-2 D614G Spike Mutation Increases Entry Efficiency with Enhanced ACE2-Binding Affinity. Nat. Comm. 12:1. doi: 10.1038/s41467-02121118-2

Public Health England [PHE] (2020). Investigation of Novel SARS-COV-2 Variant: Variant of Concern 202012/01 Technical Briefing 3. 1-11. Available online at: https://www.gov.uk/government/publications/investigation-of-novel-sarscov-2-variant-variant- of-concern- 20201201 (accessed August 2, 2020).

Resende, P. C., João, F. B., Romero, H. T., Ighor, A., Luciana, A., Ana, C. M., et al. (2021). "Severe Acute Respiratory Syndrome Coronavirus 2 P.2 Lineage Associated with Reinfection Case, Brazil, June-October 2020.”. Emerg. Infect. Dis. 27, 1789-1794. doi: 10.3201/eid2707.210401

Silva, J. C., Valtuir, B. F., Sura, A. B. F. L., Euclides, M. T. F., and Fulvio, A. S. (2021). "New Brazilian Variant of the SARS-CoV-2 (P1/Gamma) of COVID19 in Alagoas State.”. Brazil. J. Infect. Dis. 25:101588. doi: 10.1016/j.bjid.2021. 101588

Voloch, C. M., Ronaldo, S. F., Luiz, G. P. A., Cynthia, C. C., Otavio, J. B., Alexandra, L. G., et al. (2021). "Genomic Characterization of a Novel SARS-CoV-2 Lineage from Rio de Janeiro, Brazil.” Edited by Colin R. Parrish. J. Virol. 95, 1-5. doi: 10.1128/JVI.00119-21

Volz, E., Swapnil, M., Meera, C., Jeffrey, C. B., Robert, J., Susan, H., et al. (2021). Transmission of SARS-CoV-2 Lineage B.1.1.7 in England: Insights from Linking Epidemiological and Genetic Data. MedRxiv 30:20249034. doi: $10.1101 / 2020.12 .30 .20249034$

WHO (2021). “COVID-19 Weekly Epidemiological Update.”.Geneva: World Health Organization 46, 1-19.

WorldOMeter (2021). Cases of Coronavirus in Brazil 2021. Available online at: https://www.worldometers.info/coronavirus/country/brazil/ (accessed August 2, 2020).

Conflict of Interest: The authors declare that the research was conducted in the absence of any commercial or financial relationships that could be construed as a potential conflict of interest.

The reviewer AS declared a shared affiliation with one of the authors, IC, to the handling editor at the time of review.

Publisher's Note: All claims expressed in this article are solely those of the authors and do not necessarily represent those of their affiliated organizations, or those of the publisher, the editors and the reviewers. Any product that may be evaluated in this article, or claim that may be made by its manufacturer, is not guaranteed or endorsed by the publisher.

Copyright (c) 2022 Henriques-Santos, Farjun, Corrêa, Figueiredo, Fidalgo-Neto and Kuriyama. This is an open-access article distributed under the terms of the Creative Commons Attribution License (CC BY). The use, distribution or reproduction in other forums is permitted, provided the original author(s) and the copyright owner(s) are credited and that the original publication in this journal is cited, in accordance with accepted academic practice. No use, distribution or reproduction is permitted which does not comply with these terms. 